\title{
Human Performance on Insight Problem Solving: A Review
}

\author{
Yun Chu ${ }^{1}$ and James N. MacGregor ${ }^{2}$
}

Invited

\begin{abstract}
:
The article provides a review of recent research on insight problem-solving performance. We discuss what insight problems are, the different types of classic and newer insight problems, and how we can classify them. We also explain some of the other aspects that affect insight performance, such as hints, analogs, training, thinking aloud, and individual differences. In addition, we describe some of the main theoretical explanations that have been offered. Finally, we present some measures of insight and relevant neuroscience contributions to the area over the last decade.
\end{abstract}

Keyword:

insight

${ }^{1}$ State University of New York, Purchase College; ${ }^{2}$ University of Victoria 


\section{Introduction: What Is Insight?}

Most people have had the "Aha" experience when they suddenly discover the solution to a problem that, until that point, had left them baffled. Often, the experience is accompanied by a feeling of puzzlement about why the solution eluded them for so long. What caused the mental block in the first place? Cognitive scientists refer to this experience as "insight problem solving," and study the phenomenon using problems that tend to evoke this type of solution experience. It is therefore convenient to refer to such problems as "insight problems" (Smith, 1995), although this usage should not be taken to mean that every insight experience is associated with a so-called "insight problem." Individual experiences of insight may be quite idiosyncratic, and one person's insight problem may be another's commonplace task. For example, someone might experience an insight when putting together a new piece of furniture, which initially seemed to present an insurmountable challenge. To someone else, the task might proceed in a series of obvious steps that move smoothly from start to finish, with no sense of being blocked and no sudden flash of illumination. The latter process is more typical of non-insight problem solving, which typically occurs incrementally, with a conscious experience of moving toward a solution. Modern interest in insight problem solving was stimulated by an influential review that was sympathetic to the earlier Gestalt contributions (Sheerer, 1963). More recently, a seminal book on the topic of insight was The Nature of Insight by Sternberg and Davidson (1995). This review primarily focuses on significant contributions to the area of insight since the publication of that book.

Insight is important because it has been linked to creativity and scientific discoveries (Finke, 1995). It has been recognized that creative thinking aids in the solution of insight problems (Lubart \& Mouchiroud, 2003). Creativity involves divergent thinking during the initial stages of problem solving by producing and connecting various ideas, while convergent thinking synthesizes, analyzes, and verifies the ideas to generate the solution. In science, sometimes insights occur when analogical reasoning is employed (Dunbar, 1995).

What makes insight problems difficult to solve is that, initially, they often appear to be routine problems. However, a familiar solution process is the wrong approach. Take, for example, the following Matching Socks problem: single black and brown socks are in a drawer in the ratio of 5:4. How many socks do you have to pull out before you are guaranteed a pair of the same color? Initially, this appears to be a routine ratio problem, but with further thought you realize that the solution has nothing to do with the given ratio of colored socks. Before reaching what may be an insightful solution, problem solvers often get "stuck" while looking for the solution. People try everything they can think of but to no avail. At this point, they feel like they have hit a wall. People encounter impasse and stop working toward the solution, because they do not know what else they can try. 
The impasse in this problem occurs when the person thinks over and over again, "How do I use the 5:4 ratio to calculate the solution?" The problem solver overcomes impasse when she realizes it is not a ratio problem at all. The 5:4 ratio does not matter in terms of determining how many socks you have to pull out of the drawer before you obtain a pair of socks. The solution hinges on much simpler concepts: there are only two colors and socks come in pairs. To match a pair, you just need two of the same color. The problem solver may only have to run through a couple of trials in her head before arriving at the conclusion that, at most, you only need to draw three socks to match a pair. The first sock she draws is black, the second sock she draws is black, therefore, in this trial, she only needs to draw two socks to have a matching pair. An alternative outcome for a trial can be that the first sock drawn is black, but the second sock drawn is brown. The third sock drawn must be either black or brown, because there are only two colors. The third sock would complete either a black pair or a brown pair.

However, before coming to this realization and breaking impasse, the problem solver may decide to set the problem aside and attend to other matters. As a result, he may enter a period of incubation (Segal, 2004). During incubation, no conscious mental work on the problem is occurring, and participants "take a break" from thinking about it. After a period of incubation, illumination or insight might appear. What exactly occurs during incubation that leads to insight? Shanker (1995) suggested an unconscious theory of incubation. During incubation, covert mental processes work to pick out what were deemed by the conscious to be irrelevant or unusual cues in solution attainment. The unconscious then combines these ideas randomly, because the unconscious does not have as strict filters as the conscious. The solution may emerge from these mental processes. Some research has found support for this theory, while other research has found no effects of incubation. However, a meta-analysis found that different types of incubation effects depend on the type of problem and the conditions under which the problem is presented (Sio \& Ormerod, 2009).

\section{Theoretical Accounts}

During the period covered by this review, two detailed theoretical accounts of insight were proposed. To place them in perspective, we will review some of the differences in point-of-view that characterize the field. Phenomenologically, insight is often described as a sudden realization of the solution. For many, this suddenness sets insight apart as a special process when compared to non-insight problems. Metcalfe (1986) used feelingof-warmth (FOW) ratings to show the sharp increase of ratings toward the end of the process in insight problems, but not for non-insight problems. FOW ratings are explained to participants as "how warm/close do you feel you are to the solution?" However, her analyses only used the last few ratings, not the ratings for the whole problem-solving 
period. For example, in Experiment 3, the analyses took into account the last 4 ratings out of a potential 50 ratings. Each problem was allotted 5 minutes during which ratings were taken every 10 seconds. The last 4 ratings only included the last 40 seconds of the process. If insight were a gradual process, this experiment would not have been able to identify it. There is a possibility that insight is a gradual process and does not require out-of-the-ordinary processes (Weisberg, 1992). This might be especially the case with multi-step insight problems. The sudden-gradual and special-ordinary controversies are issues to consider. A further implication of the sudden appearance of solutions is that the underlying processes operate outside of conscious awareness.

Building on an earlier theoretical position (Ohlsson, 1992), Representational Change Theory (RCT) proposed that the problem solver begins with an erroneous initial representation of the problem (Knöblich et al., 1999). Any attempts in that direction will result in failure. Only when the problem solver is able to see the problem in a new light or with a new representation does the solution become attainable. The initial inappropriate representation is considered to arise because past experience prompts the construction of a problem space that does not contain the solution. The result is an impasse, which can be broken by changing the faulty representation. Two specific mechanisms of representational change were presented and tested with Matchstick Arithmetic Problems, constraint relaxation and chunk decomposition. The Matchstick Arithmetic Problem instructs the participant to move one matchstick to make the equation true. (The solution is in the Appendix.)

Figure 1. A Matchstick Arithmetic Problem.

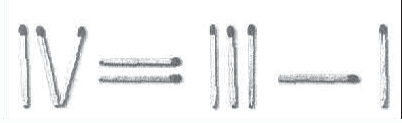

Constraint relaxation refers to the releasing of unnecessarily constraining assumptions. In the above problem, if the problem solver assumes that she can only move the matchsticks making up the roman numerals and not the operators $(+,-,=)$, she would not be able to solve the above problem. Once she realizes that the matchsticks making up the operators can also be moved, constraint relaxation occurs and she is open to finding the solution. Chunk decomposition refers to the deconstruction of perceptual chunks into smaller features, which may be recombined into potentially more productive representations. There are different levels of chunk decomposition. For example, in Matchstick Arithmetic, the easiest level of chunk decomposition is for roman numerals containing parts that have meaning on their own (I, II, and III). It is easy to separate a I from III, leaving II and I, because the separated pieces, on their own, still have meaning as roman numerals. It is a bit harder to separate the two matchsticks making up $X$, because 
$\backslash$ and / do not have meaning on their own. Since this is the case, it does not seem useful to dechunk roman numerals such as $V$ and $X$. However, if $V$ and $X$ were dechunked, then $X$ could form a $V$, and vice versa. Fewer problem solvers are willing to dechunk a $V$ and $X$ than a I, II, and III. Yet an even harder chunk decomposition is that of the operators, as it is called for in the problem above. Generally, problem solvers will not even consider moving a matchstick that is part of an operator, because operators seem unchangeable. The participant mistakenly assumes she can only move the matchsticks making up the numbers. RCT considers that both constraint relaxation and chunk decomposition occur beyond conscious, voluntary control.

A second recent theory of insight is the Criterion for Satisfactory Progress theory (CSP, formerly known as PMT_-Progress Monitoring Theory) (MacGregor, Ormerod, \& Chronicle, 2001). The theory proposes that a problem solver consciously monitors progress toward a solution against a "criterion of progress" that arises from the problem requirements. The difficulty in reaching a solution lies in the fact that, initially, the participant appears to be making sufficient progress toward the goal state. For example, take the 9-dot problem, which requires the participant to connect all 9 dots with 4 straight lines without lifting his pencil or retracing any lines. (The solution is in the Appendix.)

Figure 2. The 9-dot Problem.

There appears to be sufficient progress toward the solution as long as each line covers 2.25 dots, because 9 dots/4 lines $=2.25$ dots covered per line. Following along the edge of the perceived "square" made by the 9 dots, the first line connects 3 dots (sufficient progress) making up one side of the square. The second line connects another 3 dots (sufficient progress) on the other side of the square, even though one of the 3 dots on the second side is actually a dot that has already been covered by the first line. However, participants do not seem to count their dot coverage that way. So far, "6" dots have been covered by only 2 lines. At this point, the participant thinks she is making sufficient progress, because she still has 2 more lines to go and only 4 more dots to cover. Not until she draws the third line does she realize that the remaining fourth straight line cannot possibly cover the remaining 2 dots. The lack of lookahead ability (being able to see several moves down from the current state) leads the problem solver to think she is making sufficient progress. 
CSP has also investigated the Cheap Necklace Problem (CNP), which instructs the problem solver to make a complete closed necklace from 4 chains containing 3 links each. The participant is allowed 15 cents to do so, but it costs 2 cents to cut open a link and 3 cents to fuse it back together.

Figure 3. The Cheap Necklace Problem (CNP) in its initial state and the solution.

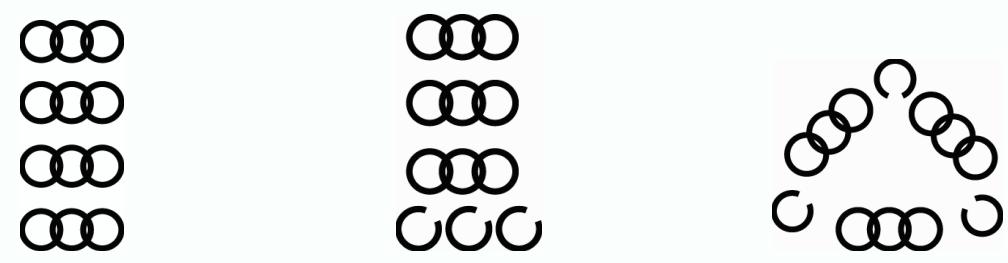

Unlike what is illustrated in Figure 3, the vast majority of participants link 2 chains (of 3 links each) end-to-end with their first 5 cents ( 2 cents to cut one link open and 3 cents to fuse it back together, once it has been linked through the loop of one of the links on other chain) for their first move (Chu, Dewald, \& Chronicle, 2007). This preferred first move appears to be making optimal progress toward the solution, because only one-third of the money has been used to connect what appears to be half of the chains ( 2 chains out of the 4). Again, the participants do not have the lookahead ability to see that starting this way will guarantee solution failure. Participants always attempt to maximize their progress toward the goal with each move. However, what may initially appear to be the best first moves will actually lead to a dead end in the problem. Participants will often persist with the same strategy over and over again, each time not being able to attain solution. As long as the criterion is being satisfied, participants do not attempt a new strategy, therefore, they cannot solve the problem. Participants often believe that there must be a trick in using the final 5 cents to complete and close the necklace. They do not stop to question that perhaps the first move needs to be re-evaluated. They seem to be fairly sure that the first move is correct, because it appears to have made so much progress on its own. If, in planning a route to a solution, none can be found that meets the current criterion of progress, then the person has arrived at an impasse, which may trigger an expansion of the search space. If this expansion results in the appearance of new possibilities that reinstate a sense of progress, then those new ideas are likely to be retained and revisited, so long as they continue to result in satisfactory progress toward the goal. These so-called "promising states" may represent partial insight. If a route appears within the planning horizon that successfully leads to the goal, then full insight has occurred.

While RCT hypothesizes automatic, unconscious processes, CSP emphasizes the role of conscious, active processes, such as planning, monitoring, and evaluating. In spite of these differences, it has been suggested that the approaches are complementary and 
might profitably be merged, since they are effective in explaining different phases of the insight process (Jones, 2003).

\section{Types of Insight Problems Used in Research}

\subsection{Classic Problems}

A varied selection of insight problems is found in a number of published sources (Ansburg \& Dominowski, 2000; Dow \& Mayer, 2004; Gilhooly \& Murphy, 2005; Metcalfe \& Wiebe, 1987; Weisberg, 1996). Dow and Mayer (2004) categorized insight problems into verbal, mathematical, and spatial.

A verbal insight problem might be:

Marsha and Marjorie were born on the same day of the same month of the same year to the same mother and the same father yet they are not twins. How is that possible?

A mathematical insight problem might be:

There are 10 bags, each containing 10 gold coins, all of which look identical. In 9 of the bags, each coin is 16 ounces, but in one of the bags, the coins are actually 17 ounces each. How is it possible, in a single weighing on an accurate weighing scale, to determine which bag contains the 17-ounce coins?

(The solutions to both problems are in the Appendix.) A spatial problem might be the 9-dot problem in Figure 2 above.

These "classic" insight problems represent the primary stimuli in insight research. Yet their availability has been limited to a small collection of spatial puzzles and verbal riddles, such as the 9-dot problem, the triangle of coins, the CNP (above), six matchsticks, and so on (Isaak \& Just, 1996), which vary widely in form, content, and level of difficulty. Typically, the relationships among them are unknown and their status as "insight problems" has, in some cases, been questioned (Weisberg, 1996). The absence of large numbers of homogenous stimuli has limited the psychological methods that can be applied in studying insight. For example, because of the lack of alternate forms of stimuli, procedures as fundamental as establishing test/retest reliability and transfer of training effects become difficult or impossible. The same stimulus limitations have restricted the applicability of neuroimaging techniques (Luo \& Knöblich, 2007). The foregoing discussion has illustrated three limitations with the problems available for studying insight. First, for most classic insight problems, it has not been established that solutions actually require insight. 
Frequently the only rationale for using a problem in an insight study is that it was used previously (Weisberg, 1996). Second, the relationships between problems are unknown. Are all insight problems members of a single class, are there identifiable subsets, or is each one unique? Without knowing the answer, it is impossible to compare results across studies that used different problems. For example, concurrent verbalization has been found to disrupt problem solving with some insight problems (Schooler, Ohlsson, \& Brooks, 1993) but not with others (Fleck \&Weisberg, 2004). This could be due to differences in the insight status of the problems used, or because they involved different types of insight problem. The current state of knowledge does not allow us to rule out either possibility. Third, even if all "classic" problems do involve insight, they are few in number and they vary widely in content, materials, mode of presentation, and level of difficulty (Luo \& Knöblich, 2007; MacGregor \& Cunningham, 2008). Until relatively recently, the field has lacked large sets of homogenous stimuli. This, in turn, has limited the kinds of research procedures that can be applied (Bowden et al., 2005). These issues will be discussed below.

\subsection{Recent Problems}

In contrast to the classic type of insight problem, several more recent sources of insight problem have been identified that promise to provide essentially unbounded sources of relatively homogenous problem. These include Matchstick Arithmetic explained above (Knöblich et al., 1999), Compound Remote Associates (CRAs) (Bowden \& Jung-Beeman, 2003a), and Rebus Puzzles (MacGregor \& Cunningham, 2008).

A CRA requires the problem solver to find the solution word associated with all words of the triad forming three compound words. For example: Age/Mile/Sand.

Rebus Puzzles combine verbal and visual or spatial cues that must be deciphered to give a common phrase or saying:

$$
\frac{\mathrm{i} \mathrm{i} \mathrm{i} \mathrm{i}}{0000}
$$

(All insight problem solutions are in the Appendix.)

In addition to providing a large repertoire of potential insight problems, these three types of problems have several useful properties. They require little or no domain-specific knowledge to solve and they are easily explained in a few minutes. More important, and in contrast to most classic problems, the experimenter can manipulate the level of difficulty of the problems. For example, for Rebus Puzzles, the more implicit assumptions that are involved, the more difficult the problem. A rebus that requires only a spatial relation principle (you just me- "just between you and $\mathrm{me}^{\prime \prime}$ ) is easier to solve than a rebus that requires two principles (SOMething — spatial relation and font property- "the start of something big"). The hardest rebuses tested have up to four principles present in one problem. Similarly, the difficulty of Matchstick Arithmetic Problems can be manipulated 
on a priori grounds (Knöblich et al., 1999), while for CRAs, empirical performance norms are available (Bowden \& Jung-Beeman, 2003b).

\subsection{Research Leading to the Classification of Problems}

How exactly are insight problems classified? There are a few ways to do this. A number of classification schemes have been proposed, some based on a priori considerations (Cunningham et al., 2010; Knöblich et al., 1999; Ohlsson, 1992), others on analysis of empirical results (Dow \& Mayer, 2004; Gilhooly \& Murphy, 2005).

Ohlsson (1992) proposed that insight problems may involve different types of restructuring, which he identified as elaboration, re-encoding, and constraint relaxation. Elaboration refers to a restructuring brought about by adding information to the original problem representation. Re-encoding involves reinterpreting the meaning of problem elements, while constraint relaxation is defined in terms of changing an initially constrained or inappropriate representation of the goal state. Ansburg and Dominowski (2000) categorized 60 verbal insight problems using this typology and found that, when they trained participants on one approach (elaboration), performance improved for all types of problem. This could have been due to the similarity between the different types of problem or to an increased performance in overall problem-solving strategies due to training. Research by Cunningham et al. (2010, p. 279) "suggests that there may be different categories of insight problems depending on the characteristics of the restructurings required to solve them. Insight problems might require: (i) changes in spatial and physical assumptions, (ii) changing defined structures and forms, (iii) misdirection, (iv) abstract and non-visualized goals, (v) number or restructuring sequences, and (vi) figure-ground type reversals... A problem's characteristics might be a reasonable way to approach the uniqueness of, and commonalities among, insight problems." While the restructuring characteristics are not the only way to classify insight problems, they might shed light on some of the various types of difficulty involved in insight problem solving.

Within a certain type of insight problem, one can also categorize the problems into differing levels of difficulty. For example, in Matchstick Arithmetic, if the solution involves moving a single matchstick that has its own meaning, the solution rates tend to be higher. Moving I from III is easier than moving / from V or X, because / has no meaning on its own. When one moves / away from V, it is said that one is relaxing constraints on the solution and decomposing perceptual chunks as mentioned above in Section 2 in regards to chunk decomposition. These two mechanisms generate specific predictions about the relative difficulty of individual problems and about differential transfer effects (Knöblich et al., 1999). Being able to have different levels of difficult allows the experimenter to manipulate the presentation of the stimuli and gather data on how it affects problem-solving performance.

Rebus Puzzles can be categorized into levels of difficulty as well. Rebus Puzzles use

- volume 3, no. 2 (Winter 2011) 
a number of different principles to encrypt a phrase or saying. For example, the rebus "thought an" (an afterthought), is solved by interpreting the relative positions of components spatially, rather than grammatically as in normal reading. In "PUNISHMENT" ("capital punishment"), the visual characteristic of the font has to be interpreted verbally, which again is not something that is done in normal reading. Thus, solving rebuses may involve relaxing one or more of the constraints that apply in processing normal text. Constraint relaxation has been considered to be an important component of insight problem solving (Ohlsson, 1992). Because the same rebus can often be represented in multiple forms, the puzzles have the potential to allow for systematic variation of one or more problem parameters. MacGregor and Cunningham (2008) used alternate forms of the same set of Rebus Puzzles to independently vary the number of constraints to be relaxed and the linguistic level at which the constraints operated (sub-word, word, or supra-word). The results indicated that both factors influence problem difficulty.

As mentioned above in Section 3.2, recent developments have uncovered three categories of problems that are candidates to satisfy the need for a pool of homogenous stimuli. These are Matchstick Arithmetic Problems (Knöblich et al., 1999), CRAs (Bowden \& Jung-Beeman, 2003a), and Rebus Puzzles (MacGregor \& Cunningham, 2008). In each case, there is evidence that some examples of the problem types are associated with insight solution, but there is also evidence that some examples are not (Knöblich et al., 1999; Bowden \& Jung-Beeman, 2003b). It is important to have either a theoretical or an empirical basis for identifying which are which. In the case of Matchstick Arithmetic Problems and Rebus Puzzles, there is also evidence that there are subcategories of problems (Knöblich et al., 1999; MacGregor \& Cunningham, 2008). Rebus Puzzles tend to be solved fairly quickly, when they are solved at all. Typically, participants are allowed 30 seconds per problem (MacGregor \& Cunningham, 2008), compared to 5 minutes or longer for the classic insight problems (Chronicle, MacGregor, \& Ormerod, 2004). The short solution durations of Rebus Puzzles may present challenges to the use of FOW ratings and protocols. If so, new or modified procedures may need to be developed.

In contrast, some studies have categorized insight problems post hoc. Dow and Mayer (2004) categorized a collection of 67 spatial, verbal, and mathematical insight problems based on participant feedback that suggested that participants viewed insight problems as being domain specific. To do so, participants were instructed to sort a number of insight problems into as many groups as they found necessary based on perceived similarities among the problems (Study 1). All possible pairs of problems were scored on the basis of how often the members of a pair were grouped together, and the scores submitted to a cluster analysis. The results indicated four main problem clusters: a spatial cluster, a verbal cluster, a mathematical cluster, and a mixed spatial-verbal cluster. Gilhooly and Murphy (2005) presented participants with 24 insight problems and 10 non-insight problems to solve. Time to solution was measured and a cluster analysis was run after dropping a few 
problems that had very low solution rates (9-dot problem, the mutilated checkerboard problem, 4 trees, and the farm problem). The analysis yielded nine clusters of problems based on performance. Some clusters belonged in the insight category and some belonged in the non-insight category. For example, cluster 1 contained insight problems such as Murples, Matching Socks, and Hole in the Earth, among others. These problems share the characteristics that they seem to be mathematical problems, but the quantities provided in the problems are useless in attaining the solution. The solution for such problems lies in visualizing the potential outcome of the situations.

In summary, there are numerous types of insight problems. Some researchers first categorize problems into insight and non-insight problems. Within the insight category, different types of insight problems have been determined using a priori and post hoc procedures. Some classic insight problems are discussed along with their drawbacks as far as the limited number of problems available and lack of problem homogeneity leading to the inability to compare insight problem performance across experiments. However, some new insight problems have come to the forefront due to their versatility. These include Rebus Puzzles, Matchstick Arithmetic Problems, and CRAs. Their advantages over classic insight problems include a large pool of problems from which to draw from and the ability to vary the level of difficulty of the problem.

\section{Theories Facilitating Insight}

A significant amount of research and theory has investigated the following aspects of insight problem solving. These include (but are not limited to) hints (Burke, Maier, \& Hoffman, 1966; Chronicle, Ormerod, \& MacGregor, 2001; Kokinov, Hadjiilieva, \& Yoveva, 1997; Ormerod, MacGregor \& Chronicle, 2002); problem analogs (Gick \& Holyoak, 1980; MacGregor, Ormerod, \& Chronicle, 2001); number of moves available (Ash \& Wiley, 2006; MacGregor, Ormerod, \& Chronicle, 2001); types of training (Ahmed \& Patrick, 2006; Ansburg \& Dominowski, 2000; Chrysikou, 2006; Cunningham \& MacGregor, 2008; Dow \& Mayer, 2004); and concurrent verbalization (Fleck \& Weisberg, 2004; Schooler, Ohlsson, \& Brooks, 1993).

\subsection{Hints Derived from Theories}

The type of hint provided for an insight problem depends on what the researcher thinks the obstacle to solution is. Chronicle, Ormerod, and MacGregor (2001) found that visual and visual-procedural hints did not help the solution to the 9-dot problem. The authors believed that the difficulty lay in the mismatch between the shape of the initial problem and the shape of the solution. Hints included shading outside of the perceived "square" formed by the nine dots. Even when the shading was in the shape of the solution lines, it did not increase solution rates significantly. Verbal hints instructing participants to pay 
attention to the shading were also provided but to no avail. Ormerod, MacGregor, and Chronicle (2002) used the 8-coin problem along with hints to aid participants in the solution process. The 8-coin problem can be presented in different initial configurations that vary the availability of preferred strategic moves. The goal is to move two coins so that each coin is touching exactly three others. (See Figure 1 below.) The solution is to stack two of the coins, which requires moving from a two-dimensional space into a three-dimensional space. Constraint relaxation of perceiving the problem as only being in two dimensions is necessary in this problem. The authors provided visual hints to the correct solution, but the hints were not noticed unless the participants' preferred strategic moves were unavailable. Therefore, the conclusion was that the participants must focus on what the constraints are before they can begin attempts in the direction of the solution. Evidently, the difficulty behind these problems was not easily resolved by providing hints present in the initial state. The question remains whether there are any ways at all to improve insight performance. Is it possible that insight problems are "hint-proof," or are there difficulties that we have not accounted for?

Figure 4. The 8-coin problem and solution.

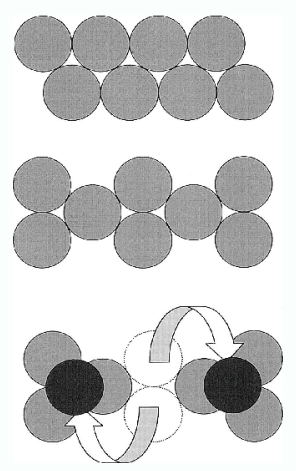

Chu, Dewald, and Chronicle (2007) found that certain hints increase solution rates. They employed a verbal hint arising from CSP Theory warning problem solvers not to perform the most common mistake in the CNP as discussed above in Section 2. The most common, yet incorrect, first move is to spend 5 cents opening a link, joining a chain to another chain end-to-end, and closing the link. This appears to be a maximizing move, because it seems that a small portion of the resources have been used to form half the necklace. However, that move guarantees failure. Thus, a verbal hint stating that the correct first move does not involve joining two chains end-to-end led to faster solutions, though not necessarily overall increased solution rates. A visual hint arising from RCT that perceptually (though not physically) "dechunked" the three links on one chain did not significantly have faster solution times than the control condition. "Dechunking" aids in obtaining the 
three individually opened links used as connectors in the solution. However, when both the verbal and visual hints were combined in one condition, this significantly increased the solution rate. This points to the fact that perhaps many constraints need to be broken in order to solve a multi-step insight problem like the CNP. Limiting the number of moves available helps solution (verbal hint). Having too many erroneous options available leads to an increased time to solution (MacGregor, Ormerod, \& Chronicle, 2001).

Thomas and Lleras (2010) gave an implicit hint by having one group swing their arms in a manner related to the solution in the two strings problem. This group was more likely to find the solution than a control group that was instructed to stretch their arms in a manner inconsistent with the solution. The authors concluded that subtly directing people's actions can aid in guiding their thoughts toward the solution. Apparently, this cue was enough to lead to higher solution rates.

\subsection{Analogical Transfer}

Sometimes providing a problem analogy improves solution rates, but often when accompanied by an explicit hint to use the analogy. Welling (2007) identified analogy as one of the main processes involved in creative thinking. There are three types of analogies. A local analogy is when an analogy is drawn based on a single characteristic that is similar between the base and the target problems. A regional analogy is mapping a set of similarities between two such problems in similar domains. A long-distance analogy is when a set of similarities is mapped between problems in completely different domains (Dunbar, 1995). Gick and Holyoak (1980) presented participants with a long-distance analogy to Duncker's Radiation Problem in the form of the Attack Dispersion story, in which a general must capture a fortress in the middle of the country. There are several roads leading to the fortress that contain mines that will detonate when a large army passes over them, but a small group of soldiers can pass over them safely. The general must think of a way to launch a full-force attack in order to capture the fortress. The solution is to send in small groups over each of the roads and have them converge at the fortress simultaneously. The participants were then presented with Duncker's Radiation Problem and instructed to use the first story to help them solve it. Although solution rates for the Radiation Problem increased significantly, the hint to use the analogy appears to have been critical to the increased success rate. Using a different base insight problem, Ormerod, Chronicle, and MacGregor (2006) reported successful spontaneous transfer from a more difficult version of the problem to a structurally similar but simpler analog. However, no corresponding transfer was observed from the simpler version to the more difficult, similar to asymmetrical transfer effects that have been observed with non-insight problems. In sum, analogical transfer occurs, but only under certain conditions. The level of similarity between the problems in an analogy plays a significant role in whether the transfer will take place.

- volume 3, no. 2 (Winter 2011) 
There are two main schools of thought on how analogical transfer happens. Reeves and Weisberg (1994) describe exemplar theories that postulate that analogical solutions come from content domain as well as the specific problems and experiences in the domain. For example, when a problem solver uses an analogy, she recalls the specific base problem that is similar to the target problem. On the other hand, both structure-mapping and pragmatic schema theories posit that deeper structural similarities are accessed when processing an analogy. That is, abstract knowledge about the problems is used to make an analogy. There is only a handful of research on the use of analogy in insight problem solving in cognitive psychology. However, there are a few more relevant articles on analogy and insight in creativity in the fields of marketing and management. Gassmann and Zeschky (2008) found that analogical thinking is necessary for the development of breakthrough innovations. Identifying a deep level of structural similarities was the key to finding solutions through analogies. Dahl and Moreau (2002) had teams of professional product designers generate ideas for a new product to facilitate eating in the car while driving. Through verbal protocol, it was found that analogy played an important role in the idea generation phase of product development. The more analogies were used, the more original the ideas when there was no external prime for a new line of products. Hints and problem analogies are two methods that have been used to influence insight solution rates.

\subsection{Theories on the Effectiveness of Training Insight}

A third method has been training, and a number of different types of training emphasizing certain aspects of problem solving have found significant results. However, despite these positive findings, questions remain as to the generalizability of training effects (Ahmed \& Patrick, 2006; Ansburg \& Dominowski, 2000; Chrysikou, 2006; Cunningham \& MacGregor, 2008; Dow \& Mayer, 2004). Ansburg and Dominowski reported five experiments testing the effects of training and/or brief instructions. Training involved practice problems that required Ohlsson's (1992) proposed elaboration mechanism to solve, while instructions stressed looking for different interpretations of a problem. The dependent variable was performance on a set of 15 verbal problems identified as requiring either elaboration or constraint relaxation to solve. In all five experiments, participants in training/instructional conditions significantly outperformed those in control conditions. Although training focused on the mechanism of elaboration, it is equally effective on problems that supposedly required constraint relaxation. Ansburg and Dominowski concluded that insightful problem solving can be construed as a general strategic thinking skill that is susceptible to training. Consistent with this conclusion are the results of Chrysikou (2006), who found that insight problem solving could be enhanced by more general training not specifically directed at solving insight problems. In this case, participants were given prior experience in placing common objects in alternative categories (the Alternative Categories Task, a 
variation of the Unusual Uses Test). Compared with the Embedded Figures Test and the Word Association Test, the Alternative Categories Test had a positive effect on subsequent performance on seven insight problems, whether or not participants were cued to the relevance of the training task, and whether or not the objects in the categorization task played a prominent role in the subsequent insight problem. The results suggest that enhancing a strategy of flexible ad hoc categorization (rather than flexible thinking more generally) improved insight problem solving across a range of problems. Both training on similar problems to the target problem and different problems to the target problem improved the performance of 12- to 13-year-olds. Better performance is attributed to teaching them how to think carefully (Adeyemo, 2003).

On the other hand, a number of studies have found the effects of training to be more specific and limited. Dow and Mayer (2004) provided participants with training in spatial, mathematical, or verbal insight problems and, in contrast to Ansburg and Dominowski's results, found that training on one type of insight problem did not generalize to other problem types (Study 3). Similarly, Cunningham and MacGregor (2008) trained participants on how to avoid or respond to impasses in insight problems. The training was provided in the context of spatial insight problems, in that it was illustrated with the 9-dot problem and followed by practice with three Matchstick Arithmetic Problems. Participants were then tested using 3 spatial problems, 6 verbal problems, and 20 Rebus Puzzles. In addition, half of the participants received the spatial and verbal problems in standard puzzlelike versions while the other half received more realistic forms of the same problem. The results showed positive training effects on solution rates for the spatial problems and Rebus Puzzles but not for verbal problems. Furthermore, the effect on spatial problems was limited to the puzzle-like versions of the problems. In this case, training based on puzzle-like spatial problems showed transfer only to other puzzle-like spatial problems or problems with a strong spatial component (Rebus Puzzles).

At the limit of specificity of transfer lies the issue of reapplying a solution or solution principle to the same problem at a subsequent point in time. While it has been argued that memory for insight solutions should be relatively impervious to decay compared to typical memory phenomena (Dominowski \& Dallob, 1996), a unique quality of some insight problems is that the solution often cannot be replicated in a later attempt. The 9-dot problem and the CNP are notorious examples of this instance. There are specific aspects of the solution that the problem solvers may remember, but the solution as a whole is not recalled. For example, in the 9-dot problem, the participant often remembers that the solution lines go outside of the perceived "square."Some participants may even remember that the solution lines form an arrow shape (Ormerod et al., 2006). However, in re-creating the solution lines, they cannot remember where the lines start, where they turn, and in what order, to satisfy the constraints of the problem. This is often the case with the CNP as well. Participants who have "stumbled" upon the solution often cannot replicate the 
process when asked to do so. Some studies have found that generating a solution results in better solution memory than being shown the solution, but Dominowski and Dallob have suggested that the critical factor in enhancing solution memory is not generating the solution per se, but having an understanding of the complete problem structure (which generating a solution may or may not require). This proposal is consistent with that of Ormerod et al., that the difficulty in replicating a solution lies in the multi-step aspect of some insight solutions preventing the solution from being encoded as a single gestalt. Some insight problems may have multiple sources of difficulty (Kershaw \& Ohlsson, 2004), and for multi-step problems, the solution may not involve just one insight or the breaking of one constraint, but the recall of many steps and the correct order and combination in which to employ them. This begs the question of what exactly is learned when solving an insight problem if you cannot recall the solution at a later time. Is a sudden leap of insight necessary, yet not sufficient, to find the solution? What conditions are necessary to transfer knowledge from one insight problem to another similar problem? When are the solution components learned well enough to be mapped onto a similar problem? (Pretz, Naples, \& Sternberg, 2003).

A very vague concept about the solution is learned when a problem solver sees the solution for the first time. She might only have understanding of some surface features of the problem. She might not yet comprehend any underlying patterns behind why the solution is such. A sudden leap of insight might be sufficient for one-step problems, such as certain matchstick problems (e.g., a matchstick solution that involves changing one of the operators). If the impasse occurred because the problem solver thought she could not change the operators, that insight alone might be sufficient to remember the solution upon future encounters. However, in multi-step problems like the 9-dot, the insight of "the lines must go outside of the 'square'"' might not be sufficient to attain solution, because the problem solver still needs to remember where the drawing of the lines starts, where the lines turn, and in what order she must draw the lines. As for transferring knowledge from one insight problem to another similar problem, it depends on what makes the problems similar, as well as how deeply the problem solver understands the solution of the base problem. For example, there are no surface similarities between Duncker's Radiation Problem and the Attack Dispersion Problem. The first one is a medical problem, while the second one is a military problem. However, if the problem solver gathers the concept that the reason why the Radiation Problem was solved is because of the deeper understanding that weaker components may converge at a single point making a much more powerful impact at the point of convergence, she reflects the deeper structural understanding needed to apply that very same concept to the Attack Dispersion Problem.

Overall, the effects of training have yielded mixed results. Some research has found that training and instructions using elaboration and constraint relaxation have improved 
performance. Other research has found that training a general ability to consider solutions outside of the normal set of options comes from a flexibility that aids insight. However, there have been other studies that found that training people in one type of insight problem (e.g., spatial) does not improve performance in a different type of insight problem. One reason could be that the different types of insight problems draw from different cognitive abilities, thus, training in one area does not affect solutions in another area. Some issues concerning training involve the generalizability of the training results. It is difficult to talk about training insight problems as a whole due to their lack of homogeneity. For example, a one-move solution for a Matchstick Arithmetic Problem is quite different than a multi-move problem such as the Cheap Necklace Problem. Especially for multi-move problems, they may require several insights to solve. In addition, memory of a solution decays. Training that requires the exactly recall of a previous problem might not be as beneficial to future problems as intended.

\subsection{Theories on Metacognition and Verbal Protocol}

One way to understand the problem solver's process is to collect a verbal protocol. The experimenter can simply ask the participant what she is thinking as she is working on the solution. Instructions are to think aloud without filters. A potential drawback of verbal protocol is verbal overshadowing. If the participant is required to explain what she is doing, this is likely to disrupt the very problem-solving process we are attempting to measure. As long as the participant focuses on the problem first and keeps verbalization as a secondary task, verbal protocol is a viable way to investigate the problem-solving process (Ericsson, 2003). Berardi-Coletta et al. $(1995$, p. 205) have found positive effects of verbal protocol due to the necessary metacognition that occurs with thinking aloud. Metacognition aids in the representation of the problem through reflection and the reassessment of the problem when one encounters obstacles (Davidson \& Sternberg, 1998). Problem solving can even benefit from appropriate probes that help people understand their own thought process (Dominowski, 1998). Fleck and Weisberg (2004) found that verbal overshadowing was not a problem in Duncker's candle problem. On the other hand, Schooler, Ohlsson, and Brooks (1993) found that verbalization hinders insight problem solving. Perhaps verbal overshadowing depends on the specific type of insight problem. Ball and Stevens (2009) presented simple and complex compound remote associates to their participants and found better performance in complex problems when they were allowed to think aloud than when they were asked to suppress talking about their thought processes. Gilhooly, Fioratou, and Henretty (2010) found more negative performance in spatial problems than verbal insight problems when participants were instructed to verbalize. There was no difference in performance between insight and non-insight problems as far as verbalization. In general, it appears that the metacognitive aspect of verbal protocol is beneficial 
for solution attainment, but this depends on the type of insight problem. Verbalization helps more in complex CRA problems and verbal insight problems than in simple CRA and spatial problems.

\subsection{Theories on Individual Differences}

Not only can characteristics of the insight problem affect performance, but so can participants' individual differences. Gilhooly and Murphy (2005) measured participants' fluid (Raven's Advanced Progressive Matrices) and crystallized intelligence, working memory (WM) capacity, short-term memory (WAIS-R digit span for verbal STM), ideational fluency and flexibility, and the central executive (sentence span). Participants were also tested on different types of insight problems (verbal and spatial) as well as non-insight problems. Gilhooly and Murphy found that figural fluency (strategic switching and inhibition) and vocabulary were correlated with performance on verbal insight problems. Figural fluency was correlated with spatial insight. Participants' fluid intelligence scores were correlated with non-insight problem performance. Therefore, insight appears to involve executive processes of switching and inhibition. The ability to change the approach or strategy while inhibiting the dominant incorrect representation of the insight problem is a necessary component in solution attainment.

Ash and Wiley (2006) found that the restructuring stage leading to insight was associated with controlled search processes. They tested participants with six insight problems and measured their working memory capacity with the Reading Span Test (RSPAN) and the Operation Span Test (OSPAN). The working memory span tests were used to measure ability to control attention. Some of the problems were Matchstick Arithmetic Problems and coin problems, among others. They found a correlation between working memory span and the ability to solve insight problems that required both the initial search space and the restructuring phase.

Chein et al. (2010) conducted three experiments using the 9-dot problem to investigate the relationship between WM capacity and insight problem-solving performance. In Experiment 1, participants were first presented with the 9-dot problem followed by a verbal WM test (OSPAN) and a spatial WM test (symmetry span or SSPAN). The participants were then categorized into high- or low-spatial WM and verbal WM. The authors not only recorded solution rate, but they also recorded whether participants drew lines outside of the "square." Even though only $14 \%$ did so, whether the participants went out of the square or not was significantly predicted by spatial WM scores. This provides support for MacGregor, Ormerod, and Chronicle's (2001) information-processing model that predicts that spatial lookahead is involved in the solution of the 9-dot problem. Verbal WM scores did not predict 9-dot performance. Since the solution rates were low, the experiment was unable to attain significance between solution rates and lookahead. Therefore, in Experiment 2, four training problems and an explicit hint to use those problems and the 
concept to extend three of the lines beyond the dots was employed to increase solution rates on the 9-dot problem. The training and hint worked, yielding a solution rate nearing $50 \%$. In this second experiment, solution rate for the 9-dot was significantly predicted by spatial WM, but not verbal WM. Solvers were significantly more likely to be in the highspatial WM group than in the low-spatial WM group. In addition, when the analyses only included solvers, individuals with higher spatial WM scores had faster solution times. One limitation of Experiment 2 is that all the training and hints might have changed the 9-dot problem so it is no longer comparable to the standard presentation of the problem (i.e., it is no longer an insight problem). Experiment 3 recorded FOW ratings for the 9-dot to ensure that it still retained its sudden solution pattern as is typical of an insight problem. The FOW pattern for the last 90 seconds for non-solvers remained flat, while the FOW pattern for solvers shot up in the last 30 seconds prior to solution attainment. This provides evidence that even with the training and hints, the 9-dot problem retained its insight characteristics. In conclusion, it appears that spatial WM capacity plays an essential role in solution attainment. Solvers were significantly more likely to have high scores in spatial WM. Individuals with high-spatial WM were also faster at solving the problem.

DeYoung, Flanders, and Peterson (2008) identified three predictors of insight performance: convergent thinking (verbal intelligence and working memory) ability, divergent thinking ability (Torrance Tests of Creative Thinking), and ability to break frame (Bruner and Postman's anomalous card task). They used nine verbal insight problems. For example, "Our basketball team won 72-49, and yet not one man scored a single point. How is that possible?" (Women's team). These types of problems are considered to be "pure" insight problems. They found that each of the three components predicted performance independently from the other two. Possessing these abilities predicts insight problem-solving performance.

To sum up all the theoretical factors facilitating insight problem solving, hints to the solution work under certain circumstances although some insight problems might require more than one hint to attain insight. Analogies may also help the solution, but it depends on the level of similarity between the base problem and the target problem. Training could improve performance, but different types of training often lead to different results. For example, training on a problem can be very specific to that problem or it can be a general ability to think in a flexible manner. Verbalization can foster successful problem solving, because it taps into metacognition that helps better understand the underlying aspects of the problem and the required mental efforts to solve the problem. However, verbalization has not helped other types of problems (e.g., spatial). Finally, individual differences in working memory capacity, specifically spatial WM span and the ability to break frame and inhibit an initial perception and switch from that perception, have been found to be related to insight performance.

- volume 3, no. 2 (Winter 2011) 


\section{Performance Measurement in Research}

The most frequently used measures are whether or not a problem is solved and the time required for solution. FOW ratings are often used to classify problems into insight/noninsight categories as mentioned above. However, FOW ratings can also be a dependent variable (Metcalfe \& Wiebe, 1987). In Experiment 4, Chu (2009) recorded FOW ratings for the CNP, the 9-dot problem, and the 8-ball problem. She also found that the better the participant's lookahead ability, the lower the FOW ratings when she approached a deadend in the Cheap Necklace Problem. Cushen and Wiley (2007) found an incremental pattern of importance ratings for important items in the problem as they approached solution and a decrease of importance ratings for items that were unimportant. Other dependent measures are insight ratings (Bowden \& Jung-Beeman, 2003b; MacGregor \& Cunningham, 2008) and neuroimaging (Bowden et al., 2005; Luo \& Niki, 2003).

There are several existing behavioral approaches to establishing the insight status of a solution, including FOW ratings mentioned above, protocol analysis (Fleck \& Weisberg, 2004), and two different methods of insight solution ratings (Bowden \& Jung-Beeman, 2003b; MacGregor \& Cunningham, 2008). FOW ratings and protocols are collected during the solution process up to the point of solution, whereas insight solution ratings are obtained after solution. This allows more than one method to be applied during a single problem-solving session. Because there may be limitations with each of these methods, it is beneficial to combine all of them in a single empirical procedure. A typical design might involve asking participants to solve a series of potential insight problems. During the solution stage, one-third of the participants provide FOW ratings, one-third, protocols, and one-third, neither. The latter condition allows a check on whether collecting FOW ratings or protocols affects solution rates or subsequent insight ratings, which the literature suggests could occur (Schooler, Ohlsson, \& Brooks, 1993; Weisberg, 1992). Following solution, participants are asked to provide ratings of the insightfulness of the solution, using existing rating methods. Participants who fail to find a solution within the time limit are shown the solution and asked to rate its insightfulness. It is expected that two or three iterations are required to test the array of potential insight problems. The results provide, for a wide range of problems, information on insight status and solution norms. They also identify the comparative usefulness of FOW, protocol analysis, and insight ratings for identifying instances where insight solutions occurred. In addition, the results indicate the problems and conditions where collecting FOW ratings and protocols interfere with solution processes. Finally, the performance data, ratings, and protocol analysis are used to assess the usefulness of current classifications of problems and to suggest improvements.

Grant and Spivey (2003) recorded participants' saccades while they solved the Radiation Problem. They found that solvers and non-solvers focused their attention on different parts of the radiation diagram. For instance, solvers spent more time looking at the skin 
around the tumor as opposed to other irrelevant parts outside of the skin or at the tumor inside the body. The authors believed that the number of crossings across the skin was similar to the path the lasers had to pass to destroy the tumor, thus the higher solution rates. An implicit method such as recoding saccades might uncover problem-solving strategies that even participants are not aware of. There might be demand characteristics when asking participants for FOW ratings, but a benefit from recording saccades is the lack of such issues.

\subsection{Neuroimaging}

Insight problems are qualitatively different from well-defined and ill-defined problems. As stated previously, insight solutions are experienced as sudden and obvious without being able to explain the processes by which the solution was attained. An insight occurs when the solver can think of new alternatives after overcoming unwarranted assumptions about the problem (Bowden et al., 2005). The solver possesses the knowledge to find the solution, yet progress on the problem often comes to a halt at a mental impasse as he is unable to attain insight (Luo, Niki, \& Phillips, 2004a). Where in the brain does insight take place? Do different areas of the brain activate for an insight problem as compare to a noninsight problem? If this were the case, then there is support for the idea that indeed insight problems are a different type of special problem requiring separate research. There are only a handful of studies investigating the neural mechanisms of insight. Luo and Niki (2003) presented 45 translated Japanese riddles to seven Chinese participants, and performed an $\mathrm{fMRI}$ scan as the answers were explained for the top 16 riddles categorized as fitting the description "I can understand this question very well and feel it is interesting, but I do not know the answer" for each participant after they had failed to understand each riddle in three minutes. The description can be classified as denoting insight. An example of a riddle is "The thing that can move heavy logs, but cannot move a nail" (a river). The Chinese participants were familiar with this type of riddle as they had often encountered them in their childhood. The researchers found widely distributed activity in the frontal, temporal, parietal, and occipital lobes during insight. The right hippocampus was more active than the left hippocampus during the explanation of the riddles, thus the right hippocampus is used for insight. Several explanations were given as to the exact role of the hippocampus, including its involvement in the formation of associations, conjunctive representation, and pattern completion. Another conclusion is that the hippocampus mediates the process of breaking mental fixation by "reorienting" the participant through abandonment of the fixation and formation of new associations.

Luo, Niki, and Phillips (2004b) investigated 15 participants with "Aha" trials evoking insight where incomprehensible sentences were followed by cues that triggered an alternate interpretation of the key idea that aided in breaking impasse. For example, the 
ambiguous sentence "His position went up because his partner's position went down" referred to a seesaw. The participants were also presented with "non-Aha" evoking trials with sentences such as "He burned the paper because the sunlight was well focused," referring to a magnifying glass. $\mathrm{fMRI}$ scans revealed activation in the anterior cingulate cortex (ACC) and the left lateral prefrontal cortex as the participants found the insight solution. Both areas have been found to mediate cognitive conflict. The researchers concluded that the ACC is involved in the insight necessary to solve problems where the dominating incorrect interpretation of the problem and the less accessible and unusual correct interpretation lead to cognitive conflicts. Thus, the ACC appears to monitor the processes among the competing options.

Mai et al. (2004) recorded high-density event-related potentials (ERPs) in 14 participants during either easy puzzles followed by a word consistent with the obvious answer (non-insight condition) or difficult puzzles followed by a word consistent with an unusual interpretation that broke the mental set, thus leading to the correct answer (insight condition). The puzzles were similar to the stimuli in Luo and Niki (2003). The results yielded strong activity and current density in the frontocentral region for the insight condition and ACC generator indicating its involvement in the breaking of mental set.

Further support is provided in Luo, Niki, and Phillips (2004b) that found ACC involvement in the solution of insight puzzles. One condition involved insight, where the participants worked on several puzzles constructed from different principles. In the non-insight condition, all the problems were constructed from the same principle taken from formal testing items, thus allowing for top-down processing by finding the general strategy employed to attain solution. The selection of puzzles for each participant in the different conditions was similar to Luo and Niki (2003) and each puzzle was presented for only 10 seconds. fMRI scans on 21 participants indicated that both conditions activated the left lateral prefrontal cortex in this between-subjects design. However, the insight condition activated more ACC than the non-insight condition. The researchers concluded that the ACC is in charge of "early warning systems" when insight problems cannot be solved by top-down processing.

Bowden, Jung-Beeman, and colleagues have investigated insight in several studies. Kounios et al. (2006) conducted two studies using CRA problems. CRA problems contained items modified from the remote-associate task (RAT). RAT was initially developed to test creativity as the solution involved drawing unusual connections between the three problem words and the solution word. For example, participants were given the words "pick," "water," and "storm." The word "ice" is the answer because it would relate to all three. The researchers chose this task to test insight, because participants' success on RAT problems reliably correlate with their success on classic insight problems, such as the two strings problem (Schooler \& Mechler, 1995). RAT is difficult, because the problem often requires the retrieval of at least one non-dominant word to find the solution. For example, the word 
"pick" might lead to the dominant response of "toothpick." However, the correct answer involves the non-dominant association of "ice" to "pick." CRA problems are insight problems, because they usually evoke an Aha! reaction when participants attain the solution and they often cannot explain how they found the solution word. Impasse and an incubation period may also be present during the solution process. Although CRA or RAT problems are not as cognitively complex as classical insight problems, they have the advantage of allowing the presentation of numerous problems during a session as each item does not take much time and the problem and answer are short enough to test within a single visual hemifield. Therefore, CRA fulfills all the requirements to be classified as an insight problem and it is a good procedure to use in neuroimaging and EEG studies.

In Experiment 1, Kounios et al. (2006) presented CRA to 19 participants. The problem solvers attempted to find the answer for 7 seconds, after which they read aloud a target word, gave an answer, and then "rated their insight experience of recognizing the solution" (p. 3). EEG recordings were observed during the "preparation" period consisting of the two-second beginning when the participant pressed the button to indicate she was ready to work on the problem and ended when the word problem was presented on the computer screen. "Insight preparation" was associated with greater neural activity peaking over mid-frontal cortex and left anterior-temporal cortex. Non-insight preparation was associated with greater neural activity peaking over the occipital cortex. "Insight preparation" was compared against the "timeout preparation" when the 30 seconds expired for each problem and the participants did not have an answer. In Experiment 2, 25 participants were scanned with $\mathrm{fMRl}$ as they solved 135 CRA problems. Participants were allowed 15 seconds for each problem. If an answer was not produced in that time, the problem was labeled "timeout."The authors found increased activation in the ACC during the "preparation" period. The ACC is first involved in cognitive-control processes that attend to dominant associations, then it shifts attention to less likely associations activated in the right anterior temporal area for insight problems. The posterior-cingulate cortex (PCC) and bilateral posterior middle/superior-temporal gyri (M/STG) also showed slightly more activity or maintained activity during preparation for insight solutions. The temporal regions are associated with semantic processing.

Bowden and Jung-Beeman (1998) performed 2 experiments with 32 participants in each experiment using CRA. When the participant failed to solve the problem, he was instructed to read aloud the word presented on the computer screen that was either the solution or an unrelated target word. The word was shown to either the left visual field (Ivf) or the right visual field (rvf). The study found greater Ivf-RH than rvf-LH priming for solved problems, while only Ivf-RH priming for unsolved problems. In addition, there was an "Ivf-RH advantage for recognizing solutions to unsolved problems" (p. 435). Therefore, the right hemisphere showed more activation of solution-relevant information than the left hemisphere involved in recognition and production of the insight solution. Jung-Beeman 
and Bowden (2000) continued the research on hemispheric differences using CRA in a series of experiments. Participants were equally quick when making decisions about the target word when priming was presented to the Ivf-RH and to the rvf-LH 3 seconds after the problem was presented. However, quicker answers were found for Ivf-RH priming than rvf-LH, 7 seconds after problem presentation. Fifteen seconds after problem presentation, only the Ivf-RH semantic activation continued to be diffuse when a target word was presented after initial failure to solution. $\mathrm{RH}$ is involved in coarse semantic coding, while $\mathrm{LH}$ is involved in fine semantic coding. Insight requires the activation of the $\mathrm{RH}$ to maintain semantic activation of unusual interpretation of words that could lead to the answer.

Bowden and Jung-Beeman (2003a) conducted a study with 44 participants using CRA with the testing procedure as described above in Kounios et al. (2006). After failure to solution, the problem solvers demonstrated more Ivf-RH priming than rvf-LH priming on problems they had rated as involving insight (i.e., they read the word faster when it was presented to the Ivf-RH). The researchers concluded that both $\mathrm{RH}$ and $\mathrm{LH}$ contribute to problem solving, but weak solution activation for insight solutions is more likely to occur in the $\mathrm{RH}$ than in the $\mathrm{LH}$. In addition, the authors suggested that people have an insight in part because they already had semantic activation that could trigger quick recognition of the solution. A previous study by Fiore and Schooler (1998) found that participants were more likely to solve a classical insight problem with hints presented to the Ivf-RH than to the rvf-LH.

Jung-Beeman et al. (2004) performed fMRI scans on 13 participants as they solved CRA problems. In Experiment 1, the participants read the problem words, indicated whether they had a solution, gave the solution, and decided whether they experienced insight on the correct solutions. Insight solutions were produced as fast as non-insight solutions. Activity for insight and non-insight problems was the same on the $\mathrm{LH}$, which was about equivalent to the non-insight activity on the $\mathrm{RH}$. However, insight solutions indicated more neural activity in the $\mathrm{RH}$ anterior superior temporal gyrus (aSTG) than non-insight solutions. Since the RH aSTG was also active during the initial presentation of the problem (along with other areas including the visual cortex), the researchers suggested the area's involvement in both the processing of the initial words and in the solution. In Experiment 2, scalp EEG recordings on 18 participants indicated "a sudden burst of high-frequency (gamma-band) neural activity" (p. 500) in the RH aSTG starting 0.3 seconds before insight solutions, confirming that insight does indeed appear suddenly. Gamma-band activity is associated with "the activation of perceptual, lexical, and semantic representations" (p. 504). The RH aSTG is involved in coarsely coded semantic integration necessary to relate various elements in the problem to each other and to the solution. The right anterior temporal region is involved in producing connections among remotely related information during the understanding of the problem. Kounios et al. (2006) compared insight and non-insight preparation for solution and found increased occipital activity associated 
with more directed visual attention for non-insight problems only. The classification for insight/non-insight problems came from the participants' subjective feeling after finding the solution.

In sum, for insight problems, the right hemisphere was implicated in insight attainment. The ACC and several prefrontal cortex areas were active during cognitive conflict during solution search. Some frontal and temporal regions (e.g., aSTG) were involved in insight solutions. The right hippocampus was also active during insight problems. Areas of the cingulate cortex were involved in insight. There is no single major area responsible for all well-defined or insight problem solving, because the complex cognitive functions performed by the brain need the integration and cooperation of numerous regions to solve a problem. Many other mechanisms are involved in problem solving, such as working memory, attention, semantic activation, monitoring, and strategy shifting after feedback, just to name a few processes. With the current neuroimaging techniques available, uncovering the neural correlates of problem solving has become a more attainable objective. Particular brain regions have already been associated with different aspects in the complicated problem-solving process and this field of interest has the potential for useful future findings. Insight is difficult to study with neuroimaging methods, because most classical insight problems, such as the 9-dot problem or the string problem, can only be presented once. After the solution is known, the researcher cannot present the problem again. Neuroimaging requires several opportunities of insight observation that are only available if numerous problems can be presented in testing blocks (e.g., CRA, Matchstick Arithmetic, and Rebus Puzzles). Another difficulty arises when we look at the complexity of insight problem solving. For example, is neuroimaging a sufficient tool to detect the different components of problem solving, such as lookahead or constructs like maximization? We have to wonder whether these aspects of problem solving are testable with imaging techniques and whether their resolution is good enough for those purposes.

\section{Future Directions and Suggestions}

What are some important open questions? There are numerous facets of insight problem solving that warrant further research in the future: individual differences, the neuroscience of insight, identifying and classifying new insight problems, ways to identify when insight has occurred, and at what levels insight is conscious, unconscious, or both at different phases of the solution process. The research literature has not been able to account for all the reasons why some people appear to be better insight problem solvers. So far, we have a few leads on what correlates with better insight (e.g., working memory capacity, divergent thinking, etc.), but there might be other individual differences that affect insight. Neuroscience has yielded some answers as far as identifying some areas of the brain that are active during the insight solution process. One future direction is to use neuroscience 
to help in the identification and classification of new insight problems. If the pattern of brain activation is different for two insight problems, then it is possible that they belong to different categories of insight. Although if two insight problems have the same brain activation pattern, it does not necessarily mean that the two problems are of the same type. There could be other differences between the problems that are not reflected in brain activation. In any case, neuroscience can certainly help in identifying and classifying insight problems. Having a large pool of varying insight problems is one of the most important necessities in expanding knowledge about insight through empirical research. Finally, the ongoing discussion regarding the conscious and unconscious levels of the process of insight should be further researched. Of course, finding ingenious ways to measure any unconscious processes might be the main difficulty as the problem solvers themselves are unable to overtly report to the researcher about them.

\section{Conclusions}

The process of insight problem solving may appear as mysterious as the insight problems themselves. Many researchers have contributed to our understanding of insight, from what the most common difficulties are in attaining solution to what exactly happens in the brain when we reach insight. One of the aspects that distinguishes insight from other types of problems is the phenomenon of sudden "Aha!"We want to know what triggers the moment of illumination, and what we can do to help along the solution. Much research has been dedicated to finding a way to approach insight with hints and analogies. The hints we present depend on what we think the difficulty is in the process. What are the major developments discussed in this paper? We have looked at different types of insight problems and how they differ from non-insight problems. We have also compiled a catalog of insight stimuli for future research. Some types of insight problems even allow the manipulation of the problems' difficulty level. The measure of the moment of insight is a tricky concept as it occurs so suddenly. We have reviewed the neuroimaging techniques that attempt to capture this process.

What is the new perspective on the integration of the recent research? What we do know is that there is no silver bullet that explains everything about insight. We have reviewed the theories associated with insight, but even together, they cannot explain every aspect of insight. If we fully understood insight, then we should be able to give enough hints and provide enough training so an insight problem can always be solved. However, that is not the case. Perhaps some solutions consist of a combination of smaller insights. This may be especially the case for multi-step problems for which even if one has seen the solution, replication may not be possible. Insight problem solving is an area with many aspects to explore. We leave it to future research in the next decade or two to uncover further explanations for the insight process. 


\section{Appendix:}

Matchstick Arithmetic Solution:

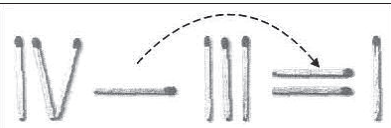

9-Dot Solution:

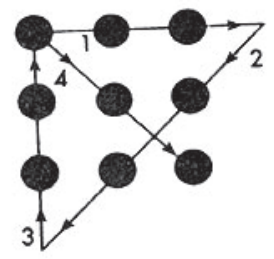

Verbal Problem Solution:

They are triplets.

Mathematical Problem Solution:

Take a different amount of coins out from each bag.

1 from the 1 st bag, 2 from the 2 nd, 3 from the 3 rd, etc.

Then weigh all those coins.

If all the bags weigh 16 ounces you will have 55 ounces $(10+9+8+7+6+5+4+3+2+1)$.

Any amount in excess of the 55 ounces will determine which bag contains the 17 ounces (two ounces over $=$ bag 2 , if it is 7 ounces over $=$ bag 7 , etc.).

Compound Remote Associates Solution:

Answer: stone

Forms the compounds STONEAGE, MILESTONE, and SANDSTONE

Rebus Solution:

Circles under eyes 


\section{References}

Adeyemo, S. A. (2003). The effect of training on insight problem solving. Psychology \& Education: An Interdisciplinary Journal, 40(2), 51-55.

Ahmed, A., \& Patrick, J. (2006). Making implicit assumptions explicit in verbal insight problem solving. Proceedings of the 28th Annual Conference of the Cognitive Science Society, pp. 955-960.

Ansburg, P. I., \& Dominowski, R. L. (2000). Promoting insightful problem solving. Journal of Creative Behaviour, 34, 30-60.

Ash, I. K., \&Wiley, J. (2006). The nature of restructuring in insight: An individual-differences approach. Psychonomic Bulletin \& Review, 13(1), 66-73.

Ball, L. J., \& Stevens, A. (2009). Evidence for a verbally-based analytic component to insight problem solving. Chapter in N. Taatgen \& H. van Rijn, J. (Eds.), Proceedings of the ThirtyFirst Annual Conference of the Cognitive Science Society. Cognitive Science Society.

Beeman, E. M., \& Bowden, E. M. (2000). The right hemisphere maintains solution-related activation for yet-to-be solved insight problems. Memory \& Cognition, 28:1231-1241.

Berardi-Coletta, B., Dominowski, R. L., Buyer, L. S., \& Rellinger, E. R. (1995). Metacognition and problem solving: A process-oriented approach. Journal of Experimental Psychology: Learning, Memory, and Cognition, 21(1), 205-223.

Bowden, E. M., \& Beeman, M. J. (1998). Getting the right idea: Semantic activation in the right hemisphere may help solve insight problems. Psychological Science, 6: 435-440.

Bowden, E. M., \& Jung-Beeman, M. (2003a). Aha! Insight experience correlates with solution activation in the right hemisphere. Psychological Bulletin Review, 10: 730-737.

Bowden, E. M., \& Jung-Beeman, M. (2003b). Normative data for 144 compound remote associates problems. Behavioral Research Methods, Instruments and Computers, 35: 634-639.

Bowden, E. M., Jung-Beeman, M., Fleck, J. I., \& Kounios, J. (2005). New approaches to demystifying insight. Trends in Cognitive Sciences, 9(7), 322-328.

Burke, R. J., Maier, N. R., \& Hoffman, R. (1966). Functions of hints in individual problemsolving. American Journal of Psychology, 79(3), 389-399.

Chein, J. M., Weisberg, R. W., Streeter, N. L., \& Kwok, S. (2010). Working memory and insight in the nine-dot problem. Memory \& Cognition, 38, 883-892.

Chronicle, E. P., MacGregor, J. N., \& Ormerod, T. C. (2004). What makes an insight problem? The role of heuristics, goal conception, and solution recoding in knowledge-lean problems. Journal of Experimental Psychology: Learning, Memory, and Cognition, 30(1), 14-27.

Chronicle, E. P., Ormerod, T. C., \& MacGregor, J. N. (2001). When insight just won't come: The failure of visual cues in the nine-dot problem. Quarterly Journal of Experimental Psychology, 54A(3), 903-919. 
Chrysikou, E.G. (2006). When shoes become hammers: Goal-derived categorization training enhances problem solving performance. Journal of Experimental Psychology: Learning, Memory, and Cognition, 33, 935-942.

Chu, Y. (2009). Human Insight Problem Solving: Performance, Processing, and Phenomenology. VDM Verlag.

Chu, Y., Dewald, A. D., \& Chronicle. E. P. (2007). Theory-driven hints in the Cheap Necklace Problem: A preliminary investigation. Journal of Problem Solving, 1(2), 18-32.

Cunningham, J. B., \& MacGregor, J. N. (2008) Training insightful problem solving: Effects of realistic and puzzle-like contexts. Creativity Research Journal, 20, 291-296.

Cunningham, J. B., MacGregor, J. N., Gibb, J., \& Harr, J. (2010). Categories of insight and their correlates: An exploration of relationships among classic-type insight problems, rebus puzzles, remote associates and esoteric analogies. Journal of Creative Behavior. (In Press).

Cushen, P. J., \& Wiley, J. (2007) Evidence for incremental restructuring in a spatial insight problem. Poster presented at the 29th Annual Conference of the Cognitive Science Society.

Dahl, D.W., \& Moreau, P. (2002). The influence and value of analogical thinking during new product ideation. Journal of Marketing Research, 39, 47-60.

Davidson, J. E. (2003). Insights about insightful problem solving. In J. E. Davidson \& R. J. Sternberg (Eds.), The Psychology of Problem Solving (pp. 149-175). Cambridge University Press.

Davidson, J. E., \& Sternberg, R. J. (1998). Smart problem solving: How metacognition helps. In D. J. Hacker, A. C. Graesser, \& J. Dunlosky (Eds.), Metacognition in Educational Theory and Practice (pp. 47-68). Erlbaum.

DeYoung, C. G., Flanders, J. L., \& Peterson, J. B. (2008). Cognitive abilities involved in insight problem solving: An individual differences model. Creativity Research Journal, 20(3), 278-290.

Dominowski, R. L. (1998). Verbalization and problem solving. In D. J. Hacker, A. C. Graesser, \& J. Dunlosky (Eds.), Metacognition in Educational Theory and Practice (pp. 25-45). Erlbaum.

Dominowski, R. L., \& Dallob, P. (1996). Insight and problem solving. In R. J. Sternberg \& J. E. Davidson (Eds.), The Nature of Insight (pp. 33-62). MIT Press.

Dow, G. T., \& Mayer, R. E. (2004). Teaching students to solve insight problems: Evidence for domain specificity in creativity training. Creativity Research Journal, 16, 389-402.

Dunbar, K. (1995). How scientists really reason: Scientific reasoning in real-world laboratories. In R. J. Sternberg \& J. E. Davidson (Eds.), The Nature of Insight (pp. 365-395). MIT Press.

Ericsson, K. A. (2003). Valid and non-reactive verbalization of thoughts during performance of tasks: Towards a solution to the central problems of introspection as a source of scientific data. Journal of Consciousness Studies, 10(9-10), 1-18. 
Finke, R. A. (1995). Creative insight and preinventive forms. In R. J. Sternberg \& J. E. Davidson (Eds.), The Nature of Insight (pp. 255-280). MIT Press.

Fiore, S. M., \& Schooler, J. W. (1998). Right hemisphere contributions to creative problem solving: Converging evidence for divergent thinking. In M. Jung-Beeman \& C. Chiarello (Eds.), Right Hemisphere Language Comprehension: Perspectives from Cognitive Neuroscience (pp. 249-371). Erlbaum.

Fleck, J. I., \& Weisberg, R. W. (2004). The use of verbal protocols as data: An analysis of insight in the candle problem. Memory \& Cognition, 32, 990-1006.

Gassmann, O., \& Zeschky, M. (2008). Opening up the solution space:The role of analogical thinking for breakthrough product innovation. Creativity and Innovation Management, 17(2), 97-106.

Gick, M. L., \& Holyoak, K. J. (1980). Analogical problem solving. Cognitive Psychology, 12, 306-355.

Gilhooly, K. J., Fioratou, E., \& Henretty, N. (2010). Verbalization and problem solving: Insight and spatial factors. British Journal of Psychology, 101(1), 81-93.

Gilhooly, K. J., \& Murphy, P. (2005) Differentiating insight from non-insight problems. Thinking and Reasoning, 11, 279-302.

Grant, E. R., \& Spivey, M. J. (2003). Eye movements and problem solving: Guiding attention guides thought. Psychological Science, 14(5), 462-466.

Isaak, M. I., \& Just, M. A. (1996). Constraints on thinking in insight and invention. In R. J. Sternberg \& J. E. Davidson, (Eds.), The Nature of Insight. MIT Press.

Jones, G. (2003). Testing two cognitive theories of insight. Journal of Experimental Psychology: Learning, Memory, and Cognition, 29(5), 1017-1027.

Jung-Beeman, M., \& Bowden, E. M. (2000). The right hemisphere maintains solution-related activation for yet-to-be-solved problems. Memory \& Cognition, 28(7), 1231-1241.

Jung-Beeman, M., Bowden, E. M., Haberman, J., Frymiare, . L., Arambel-Liu, S., Greenblatt, R., Reber, P. J., \& Kounios, J. (2004). Neural activity when people solve verbal problems with insight. PLoS Biology, 2(4), 500-510.

Kershaw, T. C., \& Ohlsson, S. (2004). Multiple causes of difficulty in insight: The case of the nine-dot problem. Journal of Experimental Psychology: Learning, Memory, and Cognition, 30(1), 3-13.

Knöblich, G., Ohlsson, S., Haider, H., \& Rhenius, D. (1999). Constraint relaxation and chunk decomposition in insight problem solving. Journal of Experimental Psychology: Learning, Memory, and Cognition, 25(6), 1534-1555.

Knöblich, G., Ohlsson, S., \& Raney, G. E. (2001). An eye movement study of insight problem solving. Memory \& Cognition, 29(7), 1000-1009.

Kokinov, B., Hadjiilieva, K. \& Yoveva, M. (1997). Is a hint always useful in problem solving? The influence of pragmatic distance on context effects. Proceedings of the 19th Annual Conference of the Cognitive Science Society, p, 974. Erlbaum. 
Kounios, J., Frymiare, J. L., Bowden, E. M., Fleck, J., Subramaniam, K., Parish, T. B., \& JungBeeman, M. (2006). The prepared mind: Neural activity prior to problem presentation predicts subsequent solution by sudden insight. Psychological Science, 17(10), 882-890.

Lubart, T. I., \& Mouchiroud, C. (2003). In J. E. Davidson \& R. J. Sternberg (Eds.), The Psychology of Problem Solving (pp. 127-148). Cambridge University Press.

Luo, J., \& Knöblich, G. (2007). Studying insight with neuroscientific methods. Methods, 42, 77-86.

Luo, J., \& Niki, K. (2003). Function of hippocampus in "insight" of problem solving. Hippocampus, 13(3), 316-323.

Luo, J., Niki, K., \& Phillips, S. (2004a). Neural correlates of the 'Aha !' reaction. NeuroReport: For Rapid Communication of Neuroscience Research, 15(13), 2013-2017.

Luo, J., Niki, K., \& Phillips, S. (2004b). The function of the anterior cingulate cortex (ACC) in the insightful solving of puzzles:The ACC is activated less when the structure of the puzzle is unknown. Journal of Psychology in Chinese Societies, 5(2), 195-213.

MacGregor, J. N., \& Cunningham, J.B. (2008). Rebus puzzles as insight problems. Behavior Research Methods, 40, 263-268.

MacGregor, J. N., Ormerod, T. C., \& Chronicle, E. P. (2001). Information processing and insight: A process model of performance on the nine-dot and related problems. Journal of Experimental Psychology: Learning, Memory, and Cognition, 27(1), 176-201.

Mai, X., Luo, J., Wu., J., \& Luo, Y. (2004). "Aha!" Effects in a guessing riddle task: An eventrelated potential study. Human Brain Mapping, 22, 261-270.

Metcalfe, J. (1986). Premonitions of insight predict impending error. Journal of Experimental Psychology: Learning, Memory, and Cognition, 12(4), 623-634.

Metcalfe, J., \&Wiebe, D. (1987). Intuition in insight and noninsight problem solving. Memory \& Cognition, 15(3), 238-246.

Novick, L. R., \& Sherman, S. J. (2003). On the nature of insight solutions: Evidence from skill differences in anagram solution. Quarterly Journal of Experimental Psychology: Human Experimental Psychology, 56A(2), 351-382.

Ohlsson, S. (1992). Information-processing explanations of insight and related phenomena. In M. Keane \& K. Gilhooly (Eds.), Advances in the Psychology of Thinking (pp. 1-44). Harvester-Wheatsheaf.

Ormerod, T. C., Fioratou, E., Chronicle, E. P., \& MacGregor, J. N. (2006). The remnants of insight. Paper presented at the 28th Annual Conference of the Cognitive Science Society (pp. 1893-1898).

Ormerod, T. C., MacGregor, J. N., \& Chronicle, E. P. (2002). Dynamics and constraints in insight problem solving. Journal of Experimental Psychology: Learning, Memory, and Cognition, 28(4), 791-799.

- volume 3, no. 2 (Winter 2011) 
Pretz, J. E., Naples, A. J., \& Sternberg, R. J. (2003). Recognizing, defining, and representing problems. In J. E. Davidson \& R. J. Sternberg (Eds.), The Psychology of Problem Solving (pp. 3-30). Cambridge University Press.

Reeves, L. M., \& Weisberg, R. W. (1994). The role of content and abstract information in analogical transfer. Psychological Bulletin, 115(3), 381-400.

Scheerer, M. (1963). Problem solving. Scientific American, 208, 118-128.

Schooler, J. W., \& Melcher, J. (1995). The ineffability of insight. In S. M. Smith, T. B. Ward, \& R. A. Finke (Eds.), The Creative Cognition Approach (pp. 249-268). MIT Press.

Schooler, J. W., Ohlsson, S., \& Brooks, K. (1993). Thoughts beyond words: When language overshadows insight. Journal of Experimental Psychology: General, 122, 166-183.

Segal, E. (2004). Incubation in insight problem solving. Creativity Research Journal, 16(1), 141-148.

Shanker, S. G. (1995). The nature of insight. Minds and Machines, 5, 561-581.

Smith, S. M. (1995). Fixation, incubation, and insight in memory, problem solving, and creativity. In S. M. Smith, T. B. Ward, \& R. A. Finke (Eds.), The Creative Cognition Approach (pp. 135-155). MIT Press.

Sio, U. N., \& Ormerod, T.C. (2009). Does incubation enhance problem solving? A metaanalysis review. Psychological Bulletin, 135, 94-120.

Sternberg, R. J., \& Davidson, J. E. (Eds.) (1995). The Nature of Insight. MIT Press.

Thomas, L. E., \& Lleras, A. (2010). Swinging into thought: Directed movement guides insight in problem solving. Psychonomic Bulletin \& Review, 16(4), 719-923.

Weisberg, R. W. (1992). Metacognition and insight during problem solving: Comment on Metcalfe. Journal of Experimental Psychology: Learning, Memory, and Cognition, 18(2), 426-431.

Weisberg, R. W. (1996). Prolegomena to theories of insight problem solving: A taxonomy of problems. In R. J. Sternberg \& J. E. Davidson (Eds.), The Nature of Insight (Paperback ed., pp. 157-196). MIT Press.

Welling, H. (2007). Four mental operations in creative cognition: The importance of abstraction. Creativity Research Journal, 19(2-3), 163-177.

Paper submitted: October 18, 2010

Paper accepted: December 12, 2010 